\title{
Cross hedging with stochastic correlation
}

\author{
Stefan Ankirchner \\ University of Bonn \\ Institute for Applied Mathematics \\ Endenicher Allee 60 \\ 53115 Bonn \\ ankirchner@hcm.uni-bonn.de
}

\author{
Gregor Heyne \\ Humboldt-Universität zu Berlin \\ Institut für Mathematik \\ Unter den Linden 6 \\ 10099 Berlin \\ heyne@math.hu-berlin.de
}

August 12, 2011

\begin{abstract}
This paper is concerned with the study of quadratic hedging of contingent claims with basis risk. We extend existing results by allowing for the correlation between the hedging instrument and the underlying of the contingent claim to be random itself. We assume that the correlation process $\rho$ evolves according to a stochastic differential equation with values between the boundaries -1 and 1 . We keep the correlation dynamics general and derive an integrability condition on the correlation process that allows to describe and compute the quadratic hedge by means of a simple hedging formula that can be directly implemented. Furthermore we show that the conditions on $\rho$ are fulfilled by a large class of dynamics. The theory is exemplified by various, explicitly given correlation dynamics.
\end{abstract}

\section{Introduction}

If a hedging instrument is not perfectly correlated with the risk to be hedged, then a nonhedgeable risk, called basis risk, remains. A prominent example for financial derivatives that entail basis risk are basket options. As an example think of options on stock market indices like the Dow Jones or the DAX. In practice such options may be hedged by trading futures or forwards written on the index. A futures on a stock index is usually highly correlated with the index itself. Figure 1 shows the daily Dax values between April and December 2008, and the daily EUREX average prices of the Dax Futures with maturity December 2008.

There are many papers dealing with optimal hedging with basis risk, see f.ex. [3] and [8] and the references therein. In the literature two different optimality criteria for the hedge have been applied so far. First, a utility based approach that aims at maximising the exponential utility of the terminal wealth minus the hedging costs (see f.ex. [1], [7], [3]). Second, a quadratic approach that aims at minimising the quadratic hedging error. In all the hedging literature concerned, the correlation between the tradable and non-tradable assets is supposed to be constant. There 


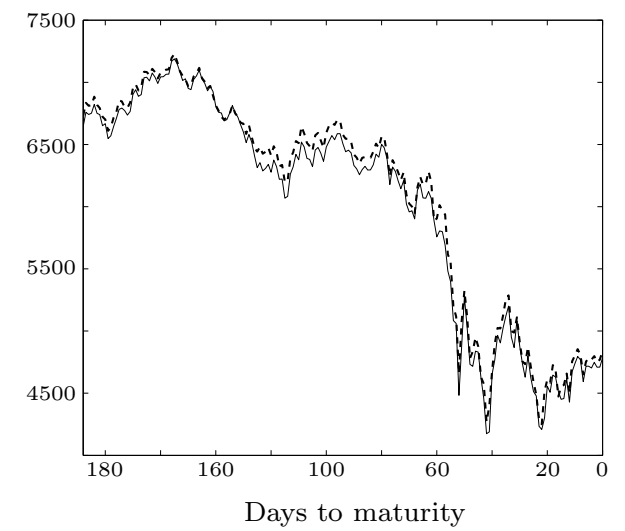

Figure 1: Daily average Dax values (continuous line) between April and December 2008 and daily EUREX average prices of the Dax Futures (dashed line) issued in April 2008 and with maturity December 2008.

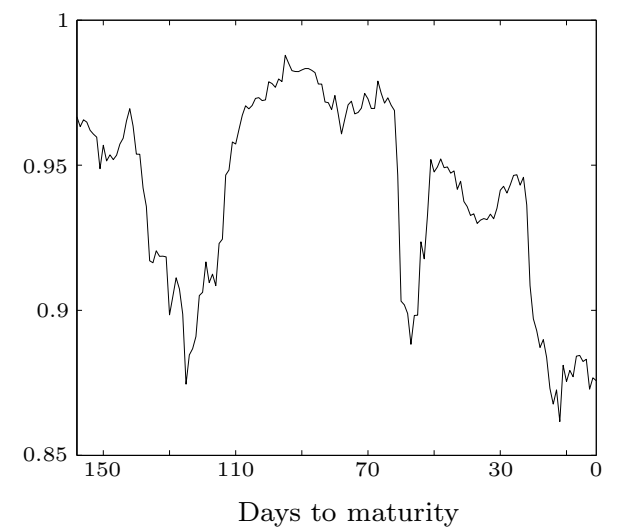

Figure 2: Correlation between the logreturns of the Dax and Dax Futures of Figure 1.

is empirical evidence, however, that often the correlation is random itself and fluctuates over time. Figure 2 shows the correlation between the Dax and EUREX Dax Futures.

In this paper we extend results on quadratic hedging with basis risk by allowing for the correlation to be random. As usual, we assume that the price of the tradable asset and the value of the non-tradable index evolve according to geometric Brownian motions. However, we will assume that the correlation between the driving Brownian motions is not constant, but a random process with values between -1 and 1 . More precisely, we will assume that the correlation process is the solution of a stochastic differential equation (SDE). This will guarantee that the correlation process possesses the Markovian property.

We consider European options on the non-tradable index and derive the asset hedging strategy that locally minimizes the quadratic hedging error, the so-called locally risk minimizing strategy (see Section 1 for an introduction into local risk minimization). Essentially, the optimal hedge can be described by the following factors: the asset hedge ratio, defined as

$$
\rho_{t} \frac{\text { index vola }}{\text { asset vola }}
$$

where $\rho_{t}$ is the correlation process, the correlation hedge ratio, defined as

$$
\gamma \frac{\text { correlation vola }}{\text { asset vola }}
$$

where $\gamma$ is the correlation between the asset and $\rho_{t}$. The derivative with respect to the asset (resp. the correlation) of the expected value of the option under the so-called minimal equivalent local martingale measure will be called asset delta (resp. correlation delta). We will show that the optimal hedge is the asset hedge ratio multiplied with the asset delta plus the correlation hedge ratio multiplied with the correlation delta, i.e.

opt. hedge $=$ asset hedge ratio $\times$ asset delta + correlation hedge ratio $\times$ correlation delta. 
In general, by assuming a stochastic correlation, there is no closed formula for the asset delta, but it is straightforward to show that it has a representation in terms of a simple expectation. The main effort, however, lies in showing that the correlation delta can be expressed as a simple expectation as-well. Under a natural integrability condition on the correlation process, we show that one may differentiate under the expectation, and hence obtains the desired representation. With this at hand, one may compute the hedging strategy by simple Monte-Carlo simulations.

It will be the topic of future research to compare the performance of the hedging formula derived with naive hedging strategies assuming constant correlation. For models with constant correlation, in [8] the performance of quadratic hedging has been compared with exponential utility based hedging as described in [11].

We want to point out to two papers that allow for stochastic correlation in pricing contingent claims: Van Emmrich [17] prices quanto options by assuming that the exchange rate is stochastically correlated with the underlying, and Frei \& Schweizer [6] deal with exponential utility indifference valuation of contingents claims based on risk sources that are stochastically correlated with assets traded on financial markets.

The paper is organised as follows. In Section 1 we give a short introdution into local risk minimization. Section 2 introduces our model and gives an overview on the main results we obtained. The details we use to derive our hedge formula are provided in Section 3. We continue in Section 4 by analyzing the boundary behaviour and integrability properties of correlation processes. We conclude with Section 5 by giving some explicit examples of correlation processes for which our main results hold.

\section{A brief review of local risk minimization}

In this section we give a short introduction into the theory of local risk minimization in a quadratic sense. The material presented is a streamlined version of [16].

We start with a filtered probability space $(\Omega, \mathcal{F}, \mathbb{P})$, where $T>0$ is a finite time horizon and the filtration $\left(\mathcal{F}_{t}\right)_{0 \leq t \leq T}$ satisfies the usual conditions, i.e. $\left(\mathcal{F}_{t}\right)_{0 \leq t \leq T}$ is right continuous and completed by the $\mathbb{P}$-null sets. We consider a financial market with one risky asset $S$ and one non-risky asset, say a money market account with dynamics $B$. We suppose that the discounted asset price $X=S / B$ is an $\mathbb{R}$-valued continuous semimartingale, and we assume that $X$ satisfies the so-called structure condition $(S C)$. This means that $X$ is a special semimartingale with canonical decomposition

$$
X=X_{0}+M+A=X_{0}+M+\int \lambda d\langle M\rangle
$$

where $M$ is a locally square integrable martingale with $M_{0}=0$, and $\lambda$ is an $\mathbb{R}$-valued, predictable process such that the mean-variance tradeoff process $K=\int_{0}^{\cdot} \lambda d A=\int_{0}^{\cdot} \lambda^{2} d\langle M\rangle$ satisfies $K_{T}<$ $\infty, \mathbb{P}$ a.s. It is well known that (SC) is related to an absence-of-arbitrage condition; see [16] for a reference. 
Definition 1.1. ([16], Definition 1.1)

The space $\Theta_{S}$ consists of all $\mathbb{R}$-valued predictable processes $\xi$ such that the stochastic integral process $\int \xi d X$ is well-defined and

$$
\mathbb{E}\left[\int_{0}^{T} \xi_{s}^{2} d\langle M\rangle_{s}+\left(\int_{0}^{T}\left|\xi_{s} d A_{s}\right|\right)^{2}\right]<\infty
$$

An $L^{2}$-strategy is a pair $\varphi=(\xi, \eta)$, where $\xi \in \Theta_{S}$ and $\eta$ is a real-valued adapted process such that the value process $V(\varphi)=\xi X+\eta$ is right-continuous and square-integrable. $\varphi$ is called 0 -achieving if $V_{T}(\varphi)=0, \mathbb{P}$-a.s.

As usual, a strategy $\varphi=(\xi, \eta)$ describes the investment decisions of an agent trading in the financial market. An investor following the strategy $\varphi$ holds $\xi_{t}$ shares of the discounted asset $X$ at time $t$, and keeps $\eta_{t}$ units in a money market account. In this section we use the money market account as numeraire so that we need not to bother about the interest rate.

We next consider a payment stream $H=\left(H_{t}\right)_{0 \leq t \leq T}$ kept fixed throughout this introduction. Mathematically, $H$ is right-continuous, adapted, real-valued and square-integrable; the interpretation is that $H_{t} \in L^{2}(\mathbb{P})$ represents the total payments on $[0, t]$ arising due to some financial contract. A European contingent claim with maturity $T$ would have $H_{t}=0$ for all $t<T$ and just an $\mathcal{F}_{T}$-measurable payoff $H_{T} \in L^{2}(\mathbb{P})$ due at time $T$; in general, the process $H$ involves both cash inflows and outlays, and can but need not be of finite variation.

Definition 1.2. ([16], Definition 1.2)

Fix a payment stream $H$. The (cumulative) cost process of an $L^{2}$-strategy $\varphi=(\xi, \eta)$ is

$$
C_{t}^{H}(\varphi)=H_{t}+V_{t}(\varphi)-\int_{0}^{t} \xi_{s} d X_{s}, \quad 0 \leq t \leq T .
$$

$\varphi$ is called self-financing (for $H$ ) if $C_{t}^{H}(\varphi)$ is constant, and mean-self-financing if $C_{t}^{H}(\varphi)$ is a martingale (which is then square-integrable). Under the assumption that $X$ fulfills the structure condition (SC) and that the mean-variance tradeoff process $K$ is continuous we say that an $L^{2}$ strategy $\varphi$ is locally risk minimizing if $\varphi$ is 0-achieving and mean-self-financing, and the cost process $C^{H}(\varphi)$ is strongly orthogonal to $M$, i.e. $\left\langle M, C^{H}(\varphi)\right\rangle_{t}=0$, for all $t \in[0, T]$.

Thus, $C_{t}(\varphi)$ comprises the hedger's accumulated costs during $[0, t]$ including the payments $H_{t}$, and $V_{t}(\varphi)$ should therefore be interpreted as the value of the portfolio $\varphi_{t}=\left(\xi_{t}, \eta_{t}\right)$ held at time $t$ after the payments $H_{t}$. In particular, $V_{T}(\varphi)$ is the value of the portfolio $\varphi_{T}$ upon settlement of all liabilities, and a natural condition is then to restrict to 0 -achieving strategies as defined in Definition 1.1.

Remark 1.3. ([16], Remark 1.3)

Observe that if $\varphi_{t}=\left(\xi_{t}, \eta_{t}\right)$ is a 0 -achieving and mean-self-financing $L^{2}$-strategy for $H$, then $\varphi$ is uniquely determined from $\xi$ (and of course $H$ ). 
It is well known that the locally risk-minimizing strategy can be obtained via the so-called Föllmer-Schweizer decomposition (FS) of the final payment $H_{T}$. That is the decomposition of $H_{T}$ into

$$
H_{T}=H_{T}^{(0)}+\int_{0}^{T} \xi_{s}^{H_{T}} d X_{s}+L_{T}^{H_{T}}, \quad \mathbb{P}-a . s,
$$

where $H_{T}^{(0)} \in L^{2}(\mathbb{P})$ is $\mathcal{F}_{0}$-measurable, $\xi^{H_{T}}$ is in $\Theta_{S}$, and the process $L^{H_{T}}$ is a (right-continuous) square-integrable martingale strongly orthogonal to $M$ and satisfying $L_{0}^{H_{T}}=0$. Notice that such a decomposition can be shown to be unique. Once we have (2), the desired strategy $\varphi=(\xi, \eta)$ is then given by

$$
\xi=\xi^{H_{T}}, \quad \eta=V^{H_{T}}-\left(\xi^{H_{T}}\right) X,
$$

with

$$
V_{t}^{H_{T}}=H_{T}^{(0)}+\int_{0}^{t} \xi_{s}^{H_{T}} d X_{s}+L_{t}^{H_{T}}-H_{t}, \quad 0 \leq t \leq T .
$$

(see Proposition 5.2 of [16]). Furthermore, the associated cost process is given by

$$
C_{t}^{H}(\varphi)=H_{T}^{(0)}+L_{t}^{H_{T}}, \quad 0 \leq t \leq T .
$$

\section{The model and the main results}

Let $W=\left(W^{1}, W^{2}, W^{3}\right)$ be a three-dimensional Brownian motion on a probability space $(\Omega, \mathcal{F}, P)$. Consider two processes with dynamics

$$
\begin{aligned}
& d S_{t}=S_{t}\left(\mu_{X} d t+\sigma_{X} d W_{t}^{1}\right) \\
& d U_{t}=U_{t}\left(\mu_{I} d t+\sigma_{I}\left(\rho_{t} d W_{t}^{1}+\sqrt{1-\rho_{t}^{2}} d W_{t}^{2}\right)\right),
\end{aligned}
$$

where $W^{1}$ and $W^{2}$ are independent Brownian motions. To simplify the presentation we will assume throughout that all coefficients are constant, more precisley $\mu_{X}, \mu_{I} \in \mathbb{R}$ and $\sigma_{X}, \sigma_{I} \in$ $\mathbb{R} \backslash\{0\}$.

We assume that $S$ is the price process of a tradable asset, and $U$ the value process of a non-tradable index. The correlation $\rho$ is assumed to follow

$$
d \rho_{t}=a\left(\rho_{t}\right) d t+g\left(\rho_{t}\right) d \hat{W}_{t}, \quad t \geq 0,
$$

where $\hat{W}$ is given by $\hat{W}=\gamma W_{u}^{1}+\delta W_{u}^{2}+\sqrt{1-\gamma^{2}-\delta^{2}} W_{u}^{3}$, with $W^{3}$ being a Brownian motion independent of $W^{1}$ and $W^{2}$, and $\gamma$ and $\delta$ real numbers such that $\delta^{2}+\gamma^{2} \leq 1$. For the moment, we assume that the coefficients of the correlation dynamics, $a$ and $g$, belong to $\mathcal{C}^{1}(-1,1)$, and that there exists a unique solution $\rho$ of $(6)$ with values in $[-1,1]$.

Throughout we suppose that the interest $r>0$ is constant, and let $B_{t}=e^{r t}$. The discounted processes $S$ and $U$ will be denoted by

$$
X_{t}=e^{-r t} S_{t}, \quad I_{t}=e^{-r t} U_{t} .
$$


Notice that

$$
\begin{aligned}
d X_{t} & =X_{t}\left(\left(\mu_{X}-r\right) d t+\sigma_{X} d W_{t}^{1}\right) \\
d I_{t} & =I_{t}\left(\left(\mu_{I}-r\right) d t+\sigma_{I}\left(\rho_{t} d W_{t}^{1}+\sqrt{1-\rho_{t}^{2}} d W_{t}^{2}\right)\right) .
\end{aligned}
$$

Consider a derivative $d\left(U_{T}\right)$ depending on the non-tradable index. Let $h(x)=e^{-r T} d\left(e^{r T} x\right)$. Then $d\left(U_{T}\right)=e^{r T} h\left(I_{T}\right)$. Our goal is to analyse how to hedge the liability $h\left(I_{T}\right)$ by trading the asset $X$.

Since the market is incomplete we need to choose a criteria according to which strategies are chosen and the prices of contingent claims are computed. We will use the framework of local risk minimization of Section 1.

Our first main result is an explicit hedge formula, which can be easily implemented, for example by simple Monte Carlo simulation. We will state it right away in Theorem 2.1, after a brief collection of some notations and assumptions.

We will need the conditional versions of the processes $I$ and $\rho$, which are given by

$$
\begin{aligned}
I_{s}^{t, y, v} & =y+\int_{t}^{s} I_{u}^{t, y, v}\left(\left(\mu_{I}-r\right) d u+\sigma_{I}\left(\rho_{u}^{t, v} d W_{u}^{1}+\sqrt{1-\left(\rho_{u}^{t, v}\right)^{2}} d W_{u}^{2}\right)\right) \\
\rho_{s}^{t, v} & =v+\int_{t}^{s} a\left(\rho_{u}^{t, v}\right) d u+\int_{t}^{s} g\left(\rho_{u}^{t, v}\right) d \hat{W}_{u},
\end{aligned}
$$

for $t \in[0, T),(y, v) \in \mathbb{R} \times(-1,1)$.

In order to find a nice representation of the quadratic hedge, we also need the dynamics of the derivatives of $I^{t, y, v}$ and $\rho^{t, v}$ with respect to the initial values $y$ and $v$. Note that the derivative with respect to $y$ of $I^{t, y, v}$ is given by $\frac{\partial}{\partial y} I^{t, y, v}=\frac{I^{t, y, v}}{y}=I^{t, 1, v}$, and obviously $\frac{\partial}{\partial y} \rho^{t, v}=0$. If the correlation process neither attains -1 nor 1 up to time $T$, then the derivatives of $I^{t, y, v}$ and $\rho^{t, v}$ with respect to $v$ are defined. Moreover, the processes $\frac{\partial}{\partial v} I^{t, y, v}$ and $\frac{\partial}{\partial v} \rho^{t, v}$, denoted by $\bar{I}^{t, y, v}$ and $\bar{\rho}^{t, v}$ respectively, solve the SDE

$$
\begin{gathered}
\bar{I}_{s}^{t, y, v}=\int_{t}^{s} \bar{I}_{u}^{t, y, v}\left(\left(\mu_{I}-r\right) d u+\sigma_{I}\left(\rho_{u}^{t, v} d W_{u}^{1}+\sqrt{1-\left(\rho_{u}^{t, v}\right)^{2}} d W_{u}^{2}\right)\right) \\
+\int_{t}^{s} I_{u}^{t, y, v} \sigma_{I}\left(\bar{\rho}_{u}^{t, v} d W_{u}^{1}-\frac{\rho_{u}^{t, v}}{\sqrt{1-\left(\rho_{u}^{t, v}\right)^{2}}} \bar{\rho}_{u}^{t, v} d W_{u}^{2}\right) \\
\bar{\rho}_{s}^{t, v}=1+\int_{t}^{s} a^{\prime}\left(\rho_{u}^{t, v}\right) \bar{\rho}_{u}^{t, v} d u+\int_{t}^{s} g^{\prime}\left(\rho_{u}^{t, v}\right) \bar{\rho}_{u}^{t, v} d \hat{W}_{u}
\end{gathered}
$$

for $s \in[t, T]$ (see Theorem 38 of Chapter $V, 7$ in [13]). Notice that the correlation boundaries -1 and 1 are not attained if and only if the stopping times $\tau^{v}=\tau^{t, v}=\inf \left\{s \geq t: \rho_{s}^{t, v} \in\{-1,1\}\right\}$ satisfy $\tau^{t, v}>T, \mathbb{P}$-a.s. We formulate this as Condition

(H1) $\quad \tau^{t, v}>T, \quad \mathbb{P}-$ a.s., for any $v \in(-1,1)$.

Notice that (H1) guarantees that $\int_{t}^{T}\left(g^{\prime}\left(\rho_{u}^{t, v}\right)\right)^{2} d u<\infty, \mathbb{P}$-a.s. and

$$
\int_{t}^{T} \frac{\left(\bar{\rho}_{s}^{t, v}\right)^{2}}{1-\left(\rho_{s}^{t, v}\right)^{2}} d u<\infty, \quad \mathbb{P}-a . s .
$$


and hence the stochastic integrals appearing in (9) are defined. Finally, for our aim of deriving an explicit representation of the quadratic hedge, we need to impose a stronger integrability condition on $\rho$ and $\bar{\rho}$ than (10). More precisely, we will assume that the following condition is satisfied

(H2) There exists a $p>1$ such that for every $t \in[0, T]$ and $v_{0} \in(-1,1)$ there exists an open neighbourhood $U \subset(-1,1)$ of $v_{0}$ such that

$$
\sup _{v \in U} \mathbb{E} \int_{t}^{T}\left|\frac{\left(\bar{\rho}_{s}^{t, v}\right)^{2}}{1-\left(\rho_{s}^{t, v}\right)^{2}}\right|^{p} d s<\infty .
$$

We are now ready to state our first main result which gives an explicit expression for the locally risk minimizing strategy in terms of expectations with respect to the measure $\tilde{\mathbb{P}}$ with density $d \tilde{\mathbb{P}} / d \mathbb{P}=\mathcal{E}\left(-\int_{0} \frac{\mu_{X}-r}{\sigma_{X}} d W^{1}\right)_{T}$, where $\mathcal{E}(\cdot)$ denotes the Doléans-Dade exponential. Note that $\tilde{\mathbb{P}}$ corresponds to the so-called minimal martingale measure, see [16].

Theorem 2.1. Suppose that the coefficients a and $g$ in the dynamics of $\rho$ are continuously differentiable on $(-1,1)$. Assume furthermore that both Conditions (H1) and (H2) are satisfied. Let $h$ be Lipschitz such that the weak derivative $h^{\prime}$ is Lebesgue-almost everywhere continuous. Then, there exists a locally risk minimizing strategy $\varphi=(\xi, \eta)$ for the derivative $h\left(I_{T}\right)$. $\xi$ is given by $\xi_{t}=\xi\left(t, I_{t}, X_{t}, \rho_{t}\right)$, where

$$
\xi(t, y, x, v)=\frac{y}{x}\left[v \frac{\sigma_{I}}{\sigma_{X}} \tilde{\mathbb{E}}\left[h^{\prime}\left(I_{T}^{t, y, v}\right) I_{T}^{t, 1, v}\right]+\frac{g(v) \gamma}{\sigma_{X}} \tilde{\mathbb{E}}\left[h^{\prime}\left(I_{T}^{t, y, v}\right) I_{T}^{t, 1, v}\right]\right] .
$$

The proof of this Theorem is postponed to Section 3.

Remark 2.2. In terms of the original processes $S$ and $U$ the hedge would be given by $\xi_{t}=$ $\hat{\xi}\left(t, U_{t}, S_{t}, \rho_{t}\right)$ where

$$
\hat{\xi}(t, y, x, v)=\frac{y}{x}\left[v \frac{\sigma_{I}}{\sigma_{X}} \tilde{\mathbb{E}}\left[d^{\prime}\left(U_{T}^{t, y, v}\right) U_{T}^{t, 1, v}\right]+\frac{g(v) \gamma}{\sigma_{X}} \tilde{\mathbb{E}}\left[d^{\prime}\left(U_{T}^{t, y, v}\right) \bar{U}_{T}^{t, 1, v}\right]\right] e^{-r(T-t)},
$$

with $\bar{U}$ obtained in the same way as $\bar{I}$.

Before we state our second main contribution, let us apply the previous result to derive the locally risk minimizing strategy for a European Call option.

Corollary 2.3. Suppose that correlation is a deterministic function of time. For strike $K>0$, let $d(x)=\max \{(x-K), 0\}$. Then, there exists a locally risk minimizing strategy $\varphi=(\xi, \eta)$ for the derivative $d\left(U_{T}\right)$. $\xi$ is given by

$$
\xi_{t}=\rho_{t} \frac{U_{t} \sigma_{I}}{S_{t} \sigma_{X}} \Delta_{B S}\left(t, U_{t}, \kappa_{t}, \sigma_{I}\right)
$$

where $\kappa_{t}=-\left(\mu_{I}-r\right)(T-t)+\sigma_{I}\left(\frac{\mu_{X}-r}{\sigma_{X}}\right) \int_{t}^{T} \rho_{s} d s$ and, with $\Phi$ the standard normal cumulative distribution function,

$$
\Delta_{B S}(t, y, q, \sigma)=\exp (-q) \Phi\left(\frac{\ln (y / K)+\left(r+\sigma_{I}^{2} / 2\right)(T-t)-q}{\sigma_{I} \sqrt{T-t}}\right)
$$


is the Black-Scholes delta for options on stocks with continuous dividend yield $q$.

The content of the preceeding Corollary is only a slight extension of a result already mentioned in [8] for the case of constant correlation. The proof is a simple straightforward calculation.

Remark 2.4. From the local risk minimizing strategy we can easily deduce the so-called meanvariance optimal hedging strategy of the payoff $h\left(I_{T}\right)$. The mean variance hedge is defined to be the self-financing strategy minimzing the variance of the global hedging error, and usually differs from the local risk minimizing strategy (see [15] for an introduction into mean-variance hedging). In the model considered here, the mean variance trade-off process is deterministic, and hence, by an appeal to Theorems 4.6 and 4.7 of Schweizer [15], the mean variance hedge has a representation allowing to derive it numerically by a simply recursion. Namely, letting $w=\tilde{\mathbb{E}}\left(h\left(I_{T}\right)\right)$, the mean-variance optimal strategy $(\tilde{\xi}, \tilde{\eta})$ for $h\left(I_{T}\right)$ satisfies, with $\tilde{\xi}=\xi^{(w)}$,

$$
\xi_{t}^{(w)}=\xi_{t}+\frac{\mu_{X}-r}{\sigma_{X}^{2} X_{t}}\left[\tilde{\mathbb{E}}\left(h\left(I_{T}\right) \mid \mathcal{F}_{t}\right)-w-\int_{0}^{t} \xi_{s}^{(w)} d X_{s}\right]
$$

for all $t \in[0, T]$ and

$$
\tilde{\eta}_{t}=w+\int_{0}^{t} \xi_{s}^{(w)} d X_{s}-\xi_{t}^{(w)} X_{t}
$$

for all $t \in[0, T]$.

Our second main result concerns conditions on the coefficients $a$ and $g$ of $\rho$ such that Conditions (H1) and (H2) are fulfilled.

Theorem 2.5. Let $a$ and $g$ be continuously differentiable with bounded derivatives. We assume that $g(-1)=g(1)=0$, and that $g$ does not have any roots in $(-1,1)$. If

$$
\limsup _{x \uparrow 1} \frac{2 a(x)(1-x)}{g^{2}(x)}<0 \text { and } \liminf _{x \downarrow-1} \frac{2 a(x)(1+x)}{g^{2}(x)}>0,
$$

then both Conditions (H1) and (H2) are satisfied, and hence, the delta hedge is given as in Theorem 2.1.

The preceeding theorem can be generalized, which will enable us to give an example in Section 5 where the derivative of $g$ is unbounded. This, however, requires a little more notation, which for ease of exposition is avoided here. See Section 4 for a proof of Theorem 2.5 and the more general Proposition 4.3.

\section{Derivation of the hedge formula}

In this section we will derive and prove the hedge formula stated in Theorem 2.1. In Subsection 3.1 we use BSDEs to derive the Föllmer-Schweizer decomposition, which is the key to obtain the 
formula (12). In 3.2 and 3.3 we elaborate on details which we need along the way. It is in those details that we need Conditions (H1) and (H2). Let us first recall the definition of a BSDE.

As in Section 2 , let $W=\left(W^{1}, W^{2}, W^{3}\right)$ be a three-dimensional Brownian motion. The filtration generated by $W$ and completed by the $P$-null sets will be denoted by $\left(\mathcal{F}_{t}\right)$. Let $T>0$ and $\xi$ be an $\mathcal{F}_{T}$-measurable random variable, and let $f: \Omega \times[0, T] \times \mathbb{R} \times \mathbb{R}^{3} \rightarrow \mathbb{R}$ be a measurable function such that for all $(y, z) \in \mathbb{R} \times \mathbb{R}^{3}$ the mapping $f(\cdot, \cdot, y, z)$ is predictable. A solution of the BSDE with terminal condition $\xi$ and generator $f$ is defined to be a pair of predictable processes $(Y, Z)$ such that almost surely we have $\int_{0}^{T}\left|Z_{s}\right|^{2} d s<\infty, \int_{0}^{T}\left|f\left(s, Y_{s}, Z_{s}\right)\right| d s<\infty$, and for all $t \in[0, T]$

$$
Y_{t}=\xi-\int_{t}^{T} Z_{s} d W_{s}+\int_{t}^{T} f\left(s, Y_{s}, Z_{s}\right) d s
$$

The solution processes $(Y, Z)$ are often shown to satisfy some integrability properties. To this end one usually verifies whether they belong to the following function spaces. Let $p \geq 1$. We denote by $\mathbb{H}^{p}\left(\mathbb{R}^{3}\right)$ the set of all $\mathbb{R}^{3}$-valued predictable processes $\zeta$ such that $E \int_{0}^{T}\left|\zeta_{t}\right|^{p} d t<\infty$, and by $\mathbb{S}^{p}(\mathbb{R})$ the set of all $\mathbb{R}$-valued predictable processes $\delta$ satisfying $E\left(\sup _{s \in[0, T]}\left|\delta_{s}\right|^{p}\right)<\infty$.

\subsection{Deriving the FS decomposition with BSDEs}

As stated in Section 2 we use the framework of local risk minimization. Accordingly, note first that $X$ satisfies the structure condition (SC), i.e. $X$ is a special semimartingale with canonical decomposition given by $M_{t}=\int_{0}^{t} \sigma_{X} X_{s} d W_{s}, \lambda_{t}=\frac{\mu_{X}-r}{\sigma_{X}^{2} X_{t}}$ and hence $K_{t}=\left(\frac{\mu_{X}-r}{\sigma_{X}}\right)^{2} t$. In order to find the FS decomposition we consider a BSDE with terminal condition $h\left(I_{T}\right)$, and driver $f$ to be specified later,

$$
Y_{t}=h\left(I_{T}\right)-\int_{t}^{T} Z_{s} d W_{s}+\int_{t}^{T} f\left(s, Y_{s}, Z_{s}\right) d s .
$$

Assume that $f$ can be chosen such that

$$
\int_{0}^{t} \xi d X_{s}=\int_{0}^{t} Z_{s}^{1} d W_{s}^{1}-\int_{0}^{t} f\left(s, Y_{s}, Z_{s}\right) d s,
$$

for all $t \in[0, T]$. Also, by using (7), we have

$$
\int_{0}^{t} \xi d X_{s}=\int_{0}^{t} \xi \sigma_{X} X_{s} d W_{s}^{1}+\int_{0}^{t} \xi X_{s}\left(\mu_{X}-r\right) d s
$$

Uniqueness of semimartingale decompositions yields that the martingale parts of (17) and (18) coincide, and therefore it must hold $\xi_{t}=\frac{Z_{t}^{1}}{\sigma_{X} X_{t}}, P \otimes \lambda$-a.s. Moreover, the driver $f$ has to satisfy

$$
f(s, y, z)=-z^{1} \frac{\mu_{X}-r}{\sigma_{X}} .
$$

Indeed, one can show that the solution of the BSDE with generator (19) provides the FS decomposition. We summarize this in the next result, which is in fact a special case of Proposition 1.1 in $[5]$. 
Lemma 3.1. The FS decomposition of $h\left(I_{T}\right)$ is given by

$$
h\left(I_{T}\right)=Y_{0}+\int_{0}^{T} \frac{Z_{s}^{1}}{\sigma_{X} X_{s}} d X_{s}+\int_{0}^{T} Z_{s}^{2} d W_{s}^{2}+\int_{0}^{T} Z_{s}^{3} d W_{s}^{3},
$$

where $\left(Y_{s}, Z_{s}\right)_{0 \leq s \leq T}$ is the solution of the BSDE (16) with generator $f$ defined as in (19).

In order to obtain a characterization of the solution of

$$
Y_{t}=h\left(I_{T}\right)-\int_{t}^{T} Z_{s} d W_{s}-\int_{t}^{T} Z_{s}^{1} \frac{\mu_{X}-r}{\sigma_{X}} d s
$$

we consider the conditional forward SDE given by (8) and the associated conditional BSDE

$$
Y_{s}^{t, y, v}=h\left(I_{T}^{t, y, v}\right)-\int_{s}^{T} Z_{u}^{t, y, v} d W_{u}-\int_{s}^{T} Z_{u}^{1, t, y, v} \frac{\mu_{X}-r}{\sigma_{X}} d u
$$

for $s \in[t, T]$. Since the BSDE (21) is linear we know by standard results, see for example [5], that

$$
Y_{s}^{t, y, v}=\mathbb{E}\left[h\left(I_{T}^{t, y, v}\right) \Gamma_{T}^{s} \mid \mathcal{F}_{s}\right]=\mathbb{E}\left[h\left(I_{T}^{t, y, v}\right) \frac{\Gamma_{T}^{0}}{\Gamma_{s}^{0}} \mid \mathcal{F}_{s}\right]
$$

where $\Gamma_{s}^{t}=\exp \left(-\frac{\mu_{X}-r}{\sigma_{X}}\left(W_{s}^{1}-W_{t}^{1}\right)-\frac{\left(\mu_{X}-r\right)^{2}}{2 \sigma_{X}^{2}}(s-t)\right)$ is the solution of

$$
d \Gamma_{s}^{t}=\Gamma_{s}^{t}\left[-\frac{\mu_{X}-r}{\sigma_{X}} d W_{s}^{1}\right], \quad \Gamma_{t}^{t}=1 .
$$

Let $\tilde{\mathbb{P}}$ be the probability measure with density $\frac{d \tilde{\mathbb{P}}}{d \mathbb{P}}=\Gamma_{T}^{0}$, and denote with $\psi$ the function defined by

$$
\psi(t, y, v)=\tilde{\mathbb{E}}\left[h\left(I_{T}^{t, y, v}\right)\right] .
$$

That the function $\psi$ is well defined and that is has first derivatives with respect to $y$ and $v$ follows from Sections 3.2 and 3.3. The value process of the solution of the BSDE (21) satisfies $Y_{s}^{t, y, v}=\psi\left(s, I_{s}^{t, y, v}, \rho_{s}^{t, v}\right)$. Our main goal is to derive the explicit hedge formula (12). With the help of Lemma 3.9 we get the following representation for $Z_{s}^{t, y, v}$.

$$
Z_{s}^{t, y, v}=\sigma\left(I_{s}^{t, y, v}, \rho_{s}^{t, v}\right)^{*}\left(\begin{array}{c}
\partial_{y} \psi\left(s, I_{s}^{t, y, v}, \rho_{s}^{t, v}\right) \\
\partial_{v} \psi\left(s, I_{s}^{t, y, v}, \rho_{s}^{t, v}\right)
\end{array}\right)
$$

where the volatility matrix $\sigma(y, v)$ is given by

$$
\sigma(y, v)=\left(\begin{array}{ccc}
y \sigma_{I} v & y \sigma_{I} \sqrt{1-v^{2}} & 0 \\
g(v) \gamma & g(v) \delta & g(v) \sqrt{1-\gamma^{2}-\delta^{2}}
\end{array}\right), \quad y \in \mathbb{R}, v \in(-1,1) .
$$

Hence we have

$$
Z_{s}^{1, t, y, v}=I_{s}^{t, y, v} \sigma_{I} \rho_{s}^{t, v} \partial_{y} \psi\left(s, I_{s}^{t, y, v}, \rho_{s}^{t, v}\right)+g\left(\rho_{s}^{t, v}\right) \gamma \partial_{v} \psi\left(s, I_{s}^{t, y, v}, \rho_{s}^{t, v}\right),
$$


i.e. the hedge formula is given by

$$
\xi(t, y, x, v)=\frac{y \sigma_{I} v \partial_{y} \psi(s, y, v)+g(v) \gamma \partial_{v} \psi(s, y, v)}{\sigma_{X} x} .
$$

Thus by plugging in the explicit representations of $\partial_{y} \psi(s, y, v)$ and $\partial_{v} \psi(s, y, v)$, given in Section 3.3 , we obtain (12), i.e. we have proven Theorem 2.1.

Remark 3.2. Note, that the approach we take by characterizing the Föllmer-Schweizer decomposition via the solution of a linear BSDE is the same as in Example 1.3 in [5]. In our model, however, the inverse of the volatility matrix of the asset processes $X$ and $I$ is unbounded and hence does not fall within the specifications of Hypothesis 1.1 in [5]. Moreover, the coefficients of the volatility matrix of the forward processes $I$ and $\rho$ associated with the BSDE do not satisfy the prerequisites of Proposition 5.9 in [5], i.e. we do not have uniformly bounded derivatives. In order to recover our hedge formula in spite of these extensions we apply the results of our Sections 3.2 to 4 .

\subsection{Differentiability with respect to the initial conditions}

In this section we want to make some remarks on the system of SDEs, given by (8) and (9), concerning existence, uniqueness, continuity and differentiablity with respect to the initial values $y$ and $v$. We also observe the following.

Lemma 3.3. Suppose that (H1) holds. Then the SDE for $\bar{I}^{t, y, v}$ in (9) has a unique solution which is given by

$$
\bar{I}_{s}^{t, y, v}=y \mathcal{E}\left(G^{t, v}\right)_{s} \int_{t}^{s} \mathcal{E}\left(G^{t, v}\right)_{u}^{-1} d H_{u}^{t, 1, v}
$$

where

$$
H_{s}^{t, y, v}=\int_{t}^{s} I_{u}^{t, y, v} \sigma_{I}\left(\bar{\rho}_{u}^{t, v} d W_{u}^{1}-\frac{\rho_{u}^{t, v}}{\sqrt{1-\left(\rho_{u}^{t, v}\right)^{2}}} \bar{\rho}_{u}^{t, v} d W_{u}^{2}\right)
$$

and

$$
G_{s}^{t, v}=\int_{t}^{s}\left(\mu_{I}-r\right) d u+\int_{t}^{s} \sigma_{I}\left(\rho_{u}^{t, v} d W_{u}^{1}+\sqrt{1-\left(\rho_{u}^{t, v}\right)^{2}} d W_{u}^{2}\right) .
$$

Proof. Due to the Assumption (H1) we can define the semimartingales $\left(H_{s}^{t, y, v}\right)_{t \leq s \leq T}$ and $\left(G_{s}^{t, v}\right)_{t \leq s \leq T}$ as above. By looking at the dynamics (9) we see immediately that $\bar{I}_{s}^{t, y, v}$ is the solution of the linear stochastic equation

$$
\bar{I}_{s}^{t, y, v}=H_{s}^{t, y, v}+\int_{t}^{s} \bar{I}_{u}^{t, y, v} d G_{u}^{t, v}
$$

The solution of (27) is given by

$$
\bar{I}_{s}^{t, y, v}=\mathcal{E}\left(G^{t, v}\right)_{s}\left(H_{t}^{t, y, v}+\int_{t}^{s} \mathcal{E}\left(G^{t, v}\right)_{u}^{-1}\left(d H_{u}^{t, y, v}-d\left\langle H^{t, y, v}, G^{t, v}\right\rangle_{u}\right)\right),
$$


(see f. ex. Chapter IX in [14]). Notice that

$$
\begin{aligned}
d\left\langle H^{t, y, v}, G^{t, v}\right\rangle_{u} & =I_{u}^{t, y, v} \sigma_{I}^{2} \bar{\rho}_{u}^{t, v} \rho_{u}^{t, v} d u-I_{u}^{t, y, v} \sigma_{I}^{2} \sqrt{1-\left(\rho_{u}^{t, v}\right)^{2}} \frac{\rho_{u}^{t, v}}{\sqrt{1-\left(\rho_{u}^{t, v}\right)^{2}}} \bar{\rho}_{u}^{t, v} d u \\
& =0 .
\end{aligned}
$$

Since $H_{t}^{t, y, v}=0$ and $H^{t, y, v}=y H^{t, 1, v}$ we obtain (26).

Remark 3.4. The process $I^{t, y, v}$ is given by

$$
I_{s}^{t, y, v}=y \exp \left[\int_{t}^{s} \sigma_{I}\left(\rho_{u}^{t, v} d W_{u}^{1}+\sqrt{1-\left(\rho_{u}^{t, v}\right)^{2}} d W_{u}^{2}\right)+\int_{t}^{s}\left(-\frac{1}{2} \sigma_{I}^{2}+\mu_{I}-r\right) d u\right] .
$$

Moreover suppose that $\int_{t}^{s} g^{\prime}\left(\rho_{u}^{t, v}\right) d \hat{W}_{u}$ is well defined, then the second SDE in (9) has a unique solution $\bar{\rho}^{t, v}$ given by

$$
\bar{\rho}_{s}^{t, v}=\mathcal{E}\left(\int_{t}^{s} g^{\prime}\left(\rho_{u}^{t, v}\right) d \hat{W}_{u}+\int_{t}^{s} a^{\prime}\left(\rho_{u}^{t, v}\right) d u\right) .
$$

Before we end this section we want to give an auxiliary result which will be used in the sequel.

Lemma 3.5. Consider two predictable processes $c^{v}$ and $d^{v}$, depending on a parameter $v \in$ $(-1,1)$. Suppose that there exists a continuous function $D:(-1,1) \rightarrow \mathbb{R}_{+}$such that $c^{v}+\left|d^{v}\right| \leq$ $D(v)$ for all $v \in(-1,1)$. Then the process

$$
b_{s}^{t, v}=\mathcal{E}\left(\int_{t}^{s} d_{u}^{v} d \hat{W}_{u}+\int_{t}^{s} c_{u}^{v} d u\right),
$$

is defined, and for all $p \geq 1$ and $v_{0} \in(-1,1)$ there exists an open neighbourhood $U \subset(-1,1)$ of $v_{0}$, such that

$$
\sup _{v \in U} \mathbb{E}\left(\sup _{t \leq u \leq T}\left|b_{u}^{t, v}\right|^{p}\right)<\infty .
$$

Proof. Let $p \geq 1$. The Burkholder-Davis-Gundy Inequality implies that for a constant $C_{p}$, depending only on $p$, we have,

$$
\begin{aligned}
\mathbb{E}\left(\sup _{t \leq u \leq T}\left|b_{u}^{t, v}\right|^{p}\right) & \leq e^{p(T-t) D(v)} \mathbb{E}\left(\sup _{t \leq u \leq T}\left|\mathcal{E}\left(\int_{t}^{u} d_{w}^{v} d \hat{W}_{w}\right)\right|^{p} \mid\right) \\
& \leq C_{p} e^{p(T-t) D(v)} \mathbb{E}\left(\left.\left.\left|\int_{t}^{T}\right|\left(d_{u}^{v}\right)^{2} \mathcal{E}\left(\int_{t}^{u} d_{w}^{v} d \hat{W}_{w}\right)\right|^{2} d u\right|^{p / 2}\right) \\
& \leq C_{p} e^{p(T-t) D(v)} D^{p}(v) \mathbb{E}\left(\left.\left.\left|\int_{t}^{T}\right| \mathcal{E}\left(\int_{t}^{u} d_{w}^{v} d \hat{W}_{w}\right)\right|^{2} d u\right|^{p / 2}\right)
\end{aligned}
$$

In the rest of the proof we have to distinguish between $p \geq 2$ and $p \in[1,2)$. We first consider $p \geq 2$. By Jensen's inequality and Fubini's theorem we get

$$
\mathbb{E}\left(\left.\left.\left|\int_{t}^{T}\right| \mathcal{E}\left(\int_{t}^{u} d_{w}^{v} d \hat{W}_{w}\right)\right|^{2} d u\right|^{p / 2}\right) \leq \mathbb{E}\left(\int_{t}^{T}\left|\mathcal{E}\left(\int_{t}^{u} d_{w}^{v} d \hat{W}_{w}\right)\right|^{p} d u\right)=\int_{t}^{T} \mathbb{E}\left(\left|\mathcal{E}\left(\int_{t}^{u} d_{w}^{v} d \hat{W}_{w}\right)\right|^{p}\right) d u
$$


Notice that $\left|\mathcal{E}\left(\int_{t}^{u} d_{w}^{v} d \hat{W}_{w}\right)\right|^{p}=\exp \left(\int_{t}^{u} p d_{w}^{v} d \hat{W}_{w}+\int_{t}^{u} p^{2}\left(d_{w}^{v}\right)^{2} d w-\int_{t}^{u} p^{2}\left(d_{w}^{v}\right)^{2} d w-\frac{p}{2} \int_{t}^{u}\left(d_{w}^{v}\right)^{2} d w\right)$, and thus Hölder's Inequality implies that the left hand side of Inequality (30) can be further estimated against

$$
\begin{aligned}
\mathbb{E}\left(\left.\left.\left|\int_{t}^{T}\right| \mathcal{E}\left(\int_{t}^{u} d_{w}^{v} d \hat{W}_{w}\right)\right|^{2} d u\right|^{p / 2}\right) \\
\leq \int_{t}^{T}\left[\mathbb{E}\left(\exp \left(\int_{t}^{u} 2 p d_{w}^{v} d \hat{W}_{w}-\frac{1}{2} \int_{t}^{u} 4 p^{2}\left(d_{w}^{v}\right)^{2} d w\right)\right)\right]^{1 / 2} \\
\quad \times\left[\mathbb{E}\left(\exp \left(\int_{t}^{u} 2 p^{2}\left(d_{w}^{v}\right)^{2} d w-p \int_{t}^{u}\left(d_{w}^{v}\right)^{2} d w\right)\right)\right]^{1 / 2} d u \\
\leq \int_{t}^{T} e^{p^{2}(T-t) D(v)}\left[\mathbb{E}\left(\mathcal{E}\left(\int_{t}^{u} 2 p d_{w}^{v} d \hat{W}_{w}\right)\right)\right]^{1 / 2} d u \\
=(T-t) e^{p^{2}(T-t) D(v)},
\end{aligned}
$$

which yields

$$
\mathbb{E}\left(\sup _{t \leq u \leq T}\left|b_{u}^{t, v}\right|^{p}\right) \leq C_{p} D^{p}(v)(T-t) e^{\left(p+p^{2}\right)(T-t) D(v)},
$$

from where we deduce the result for $p \geq 2$. For $1 \leq p<2$ we use Jensen's inequality to obtain

$$
\mathbb{E}\left(\sup _{t \leq u \leq T}\left|b_{u}^{t, v}\right|^{p}\right) \leq C \mathbb{E}\left(\left.\left|\int_{t}^{T}\right| \mathcal{E}\left(\int_{t}^{u} d_{w}^{t, v} d \hat{W}_{w}\right)\right|^{2} d u \mid\right)^{p / 2}
$$

and continue with the same arguments as for $p>2$. Hence the result.

Remark 3.6. Since $I^{t, y, v}$ is lognormally distributed, independent of $v$, we have

$$
\sup _{v \in(-1,1)} \mathbb{E}\left(\left|I_{s}^{t, y, v}\right|^{p}\right)<\infty
$$

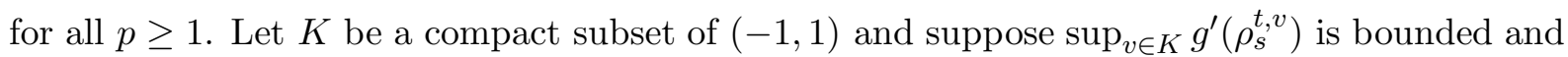
$\sup _{v \in K} a^{\prime}\left(\rho_{s}^{t, v}\right)$ is bounded above, uniformly for all $t \leq s \leq T$. Then we have $\sup _{v \in K} \mathbb{E}\left(\int_{t}^{T}\left|\bar{\rho}_{s}^{t, v}\right|^{p} d s\right)<$ $\infty$, for all $p \geq 1$, by Lemma 3.5.

\subsection{Differentiability of $\psi$}

In order to derive the hedge formula (25) we need to ensure that $\psi$ defined in (23) is continuously differentiable with respect to $v$ and $y$. We only consider the differentiablity in $v$, since for $y$ it is comparatively simpler. Since we want to use uniform integrability this is where Conditions (H1) and (H2) come into play.

Lemma 3.7. Suppose Conditions (H1) and (H2) hold. Then for all $v_{0} \in(-1,1)$ there exists an open neighbourhood $U \subset(-1,1)$ such that

$$
\sup _{v \in U} \mathbb{E}\left(\left|\bar{I}_{T}^{t, y, v}\right|^{p^{\prime}}\right)<C,
$$


for all $p^{\prime} \in[1, p)$, with $p$ of Assumption (H2). Moreover $C$ is a constant that depends only on $p$ from Condition (H2), the model parameters and $U$.

Proof. Let $p^{\prime} \geq 1$ such that $p>p^{\prime}$ with $p$ of Assumption (H2). Let $G^{t, v}$ and $H^{t, y, v}$ be defined as in Lemma 3.3. Notice that $G^{t, v}$ is lognormally distributed. Since the distribution does not depend on the correlation, there exists a constant $C \in \mathbb{R}_{+}$such that we have $\mathbb{E}\left(\left|\mathcal{E}\left(G^{t, v}\right)\right|^{2 p^{\prime}}\right) \leq C$ for all $v \in(-1,1)$. The Cauchy-Schwarz Inequality, the Burkholder-Davis-Gundy Inequality and Jensen's inequality imply

$$
\begin{aligned}
\mathbb{E}\left(\left|I_{T}^{t, y, v}\right|^{p^{\prime}}\right) & =\mathbb{E}\left(\left|\mathcal{E}\left(G^{t, v}\right)_{T} \int_{t}^{T} \mathcal{E}\left(G^{t, v}\right)_{u}^{-1} d H_{u}^{t, y, v}\right|^{p^{\prime}}\right) \\
& \leq \sqrt{C}\left(\mathbb{E}\left(\sup _{t \leq s \leq T}\left|\int_{t}^{s} \mathcal{E}\left(G^{t, v}\right)_{u}^{-1} d H_{u}^{t, y, v}\right|^{2 p^{\prime}}\right)\right)^{1 / 2} \\
& \leq \sqrt{C} C_{p^{\prime}}\left(\mathbb{E}\left(\left|\int_{t}^{T} \mathcal{E}\left(G^{t, v}\right)_{u}^{-2}\left(I_{u}^{t, y, v}\right)^{2} \sigma_{I}^{2}\left[\frac{\left(\bar{\rho}_{u}^{t, v}\right)^{2}}{1-\left(\rho_{u}^{t, v}\right)^{2}}\right] d u\right|^{p^{\prime}}\right)\right)^{1 / 2} \\
& \leq \sqrt{C} C_{p^{\prime}}\left(\mathbb{E}\left(\left|\int_{t}^{T} \mathcal{E}\left(G^{t, v}\right)_{u}^{-2 p^{\prime}}\left(I_{u}^{t, y, v}\right)^{2 p^{\prime}} \sigma_{I}^{2 p^{\prime}}\left[\frac{\left(\bar{\rho}_{u}^{t, v}\right)^{2}}{1-\left(\rho_{u}^{t, v}\right)^{2}}\right]^{p^{\prime}} d u\right|\right)\right)^{1 / 2} .
\end{aligned}
$$

Now choose $\hat{p}>1$, such that $\hat{p} p^{\prime}<p$. An application of the Hölder Inequality yields, with $\hat{q}=\frac{\hat{p}}{\hat{p}-1}$,

$$
\left.\left(\mathbb{E}\left(\left|I_{T}^{t, y, v}\right|^{p^{\prime}}\right)\right)^{2} \leq C C_{p^{\prime}}^{2}\left(\mathbb{E}\left(\left|\int_{t}^{T} \mathcal{E}\left(G^{t, v}\right)_{u}^{-2 p^{\prime} \hat{q}}\left(I_{u}^{t, y, v}\right)^{2 p^{\prime} \hat{q}} d u\right|\right)\right)^{1 / \hat{q}} \times\left(\mathbb{E}\left(\mid \int_{t}^{T} \frac{\left(\bar{\rho}_{u}^{t, v}\right)^{2}}{1-\left(\rho_{u}^{t, v}\right)^{2}}\right]^{p^{\prime} \hat{p}} d u \mid\right)\right)^{1 / \hat{p}} .
$$

For any $U \subset(-1,1)$ the supremum $\sup _{v \in U} \mathbb{E}\left(\left|\int_{t}^{T} \mathcal{E}\left(G^{t, v}\right)_{u}^{-2 p^{\prime} \hat{q}}\left(I_{u}^{t, y, v}\right)^{2 p^{\prime} \hat{q}} d u\right|\right)$ is finite due to the lognormal distribution of $I^{t, y, v}$ and the normal distribution of $G^{t, v}$, the distributions of which do not depend on the correlation process $\rho$. Therefore, with $U$ from Assumption (H2), we get $\sup _{v \in U} \mathbb{E}\left(\left|\bar{I}_{T}^{t, y, v}\right|^{p^{\prime}}\right)<C$.

The following lemma states conditions under which $\psi$ is differentiable with respect to $v$.

Lemma 3.8. Let $h$ be Lipschitz such that the weak derivative $h^{\prime}$ is Lebesgue-almost everywhere continuous. Under the Conditions (H1) and (H2) $\psi(t, y, v)$ is continuously differentiable with respect to $v$ and the partial derivative $\partial_{v} \psi(t, y, v)$ is given by

$$
\partial_{v} \psi(t, y, v)=\tilde{\mathbb{E}}\left[h^{\prime}\left(I_{T}^{t, y, v}\right) \bar{I}_{T}^{t, y, v}\right] .
$$

Proof. Let $v_{0}$ be an element of $(-1,1)$, and $p>1$ as in Condition (H2). According to Lemma 3.7 we can choose a real $\delta>0$ with $\left(v_{0}-\delta, v_{0}+\delta\right) \subset(-1,1)$ such that for all $p^{\prime} \in[1, p)$ we have $\sup _{v \in\left(v_{0}-\delta, v_{0}+\delta\right)} \mathbb{E}\left(\left|\bar{I}_{T}^{t, y, v}\right|^{p^{\prime}}\right)<\infty$. For all $v \in\left(v_{0}-\delta, v_{0}+\delta\right)$ we will show

1. $\psi(t, y, v)$ is well defined,

2. $h\left(I_{T}^{t, y, v}\right)$ is absolutely continuous in $v$, 
3. $\tilde{\mathbb{E}}\left[h^{\prime}\left(I_{T}^{t, y, v}\right) \bar{I}_{T}^{t, y, v}\right]$ is continuous at $v=v_{0}$, and

4. $\tilde{\mathbb{E}}\left[\int_{-\delta}^{\delta}\left|h^{\prime}\left(I_{T}^{t, y, v_{0}+u}\right) \bar{I}_{T}^{t, y, v_{0}+u}\right| d u\right]<\infty$.

By standard arguments these four statements imply the result (see f. ex. [4]).

The properties of $h$ imply that there exists a constant $C \in \mathbb{R}_{+}$such that $|h(x)| \leq C(1+|x|)$, and hence with Remark 3.6 we have, with $q=\frac{p}{p-1}$,

$$
\begin{aligned}
\tilde{\mathbb{E}}\left[\left|h\left(I_{T}^{t, y, v}\right)\right|\right] & \leq \mathbb{E}\left(\left|\Gamma_{T}^{0}\right|^{q}\right)^{1 / q} \mathbb{E}\left(\left|h\left(I_{T}^{t, y, v}\right)\right|^{p}\right)^{1 / p} \\
& \leq \mathbb{E}\left(\left|\Gamma_{T}^{0}\right|^{q}\right)^{1 / q} 2 C\left(1+\mathbb{E}\left|I_{T}^{t, y, v}\right|^{p}\right)^{1 / p} \\
& <\infty,
\end{aligned}
$$

and therefore $\psi$ is well defined.

Since $h$ is Lipschitz, it is absolutely continuous. Besides, $I_{T}^{t, y, v}$ is differentiable and continuous in $v$ (see Section 2), and consequently, the composition $h\left(I_{T}^{t, y, v}\right)$ is absolute continuous in $v$. With the Hölder inequality we have, for $p^{\prime} \in[1, p)$ and $\hat{p}>1$ such that $p>p^{\prime} \hat{p}>1$,

$$
\tilde{\mathbb{E}}\left[\left|h^{\prime}\left(I_{T}^{t, y, v}\right) \bar{I}_{T}^{t, y, v}\right|^{p^{\prime}}\right] \leq C\left(\mathbb{E}\left[\left|I_{T}^{t, y, v}\right|^{p^{\prime} \hat{p}}\right]\right)^{1 / \hat{p}}
$$

Thus, by Lemma 3.7 we get

$$
\sup _{v \in\left(v_{0}-\delta, v_{0}+\delta\right)} \tilde{\mathbb{E}}\left[\left|h^{\prime}\left(I_{T}^{t, y, v}\right) \bar{I}_{T}^{t, y, v}\right|^{p^{\prime}}\right] \leq C<\infty .
$$

Hence the family of random variables $\left(h^{\prime}\left(I_{T}^{t, y, v}\right) \bar{I}_{T}^{t, y, v}\right)_{v \in\left(v_{0}-\delta, v_{0}+\delta\right)}$ is uniformly integrable with respect to $\tilde{\mathbb{P}}$. Now let $\left(v_{n}\right)_{n \in \mathbb{N}}$ be any sequence in $\left(v_{0}-\delta, v_{0}+\delta\right)$ with limit $v_{0}$. Then by continuity of $h^{\prime}\left(I_{T}^{t, y, v}\right) \bar{I}_{T}^{t, y, v}$ and the properties of uniform integrability we get

$\lim _{n \rightarrow \infty}\left|\tilde{\mathbb{E}}\left[h^{\prime}\left(I_{T}^{t, y, v_{n}}\right) \bar{I}_{T}^{t, y, v_{n}}\right]-\tilde{\mathbb{E}}\left[h^{\prime}\left(I_{T}^{t, y, v_{0}}\right) \bar{I}_{T}^{t, y, v_{0}}\right]\right| \leq \lim _{n \rightarrow \infty} \tilde{\mathbb{E}}\left[\left|h^{\prime}\left(I_{T}^{t, y, v_{n}}\right) \bar{I}_{T}^{t, y, v_{n}}-h^{\prime}\left(I_{T}^{t, y, v_{0}}\right) \bar{I}_{T}^{t, y, v_{0}}\right|\right]=0$,

i.e. the continuity of $\tilde{\mathbb{E}}\left[h^{\prime}\left(I_{T}^{t, y, v}\right) \bar{I}_{T}^{t, y, v}\right]$ at $v=v_{0}$. We use boundedness of $h^{\prime}$ and Fubini's Theorem to get

$$
\begin{aligned}
\tilde{\mathbb{E}}\left[\int_{-\delta}^{\delta}\left|h^{\prime}\left(I_{T}^{t, y, v_{0}+u}\right) \bar{I}_{T}^{t, y, v_{0}+u}\right| d u\right] & \leq C \int_{-\delta}^{\delta} \mathbb{E}\left|\bar{I}_{T}^{t, y, v_{0}+u}\right| d u \\
& \leq C \sup _{v \in\left(v_{0}-\delta, v_{0}+\delta\right)} \mathbb{E}\left|\bar{I}_{T}^{t, y, v}\right|,
\end{aligned}
$$

which is finite by Lemma 3.7. Since we verified 1. -4 . the proof of Lemma 3.8 is complete.

\subsection{The hedge as variational derivative}

The control process $Z^{t, y, v}$ of the linear BSDE (21) has a representation in terms of the gradient of $\psi$ and the matrix-valued function defined by

$$
\sigma(y, v)=\left(\begin{array}{ccc}
y \sigma_{I} v & y \sigma_{I} \sqrt{1-v^{2}} & 0 \\
g(v) \gamma & g(v) \delta & g(v) \sqrt{1-\gamma^{2}-\delta^{2}}
\end{array}\right), \quad y \in \mathbb{R}, v \in(-1,1) .
$$


Lemma 3.9. Assume that (H1) and (H2) hold, that a and $g$ are continuously differentiable and let $h$ be Lipschitz such that the weak derivative $h^{\prime}$ is Lebesgue-almost everywhere continuous. Then

$$
Z_{s}^{t, y, v}=\sigma\left(I_{s}^{t, y, v}, \rho_{s}^{t, v}\right)^{*}\left(\begin{array}{l}
\partial_{y} \psi\left(s, I_{s}^{t, y, v}, \rho_{s}^{t, v}\right) \\
\partial_{v} \psi\left(s, I_{s}^{t, y, v}, \rho_{s}^{t, v}\right)
\end{array}\right)
$$

Proof. By Lemma 3.8, $\psi(s, y, v)$ is continuously differentiable in $y$ and $v$. As is shown in the fundamental Theorem 5.2 in [9], considering also Remark 5.3.i of [9], this is sufficient for the relationship (33) to hold.

Note that in [9] Imkeller, Rï $i \frac{1}{2}$ veillac, Richter establish relations as in (33) by using only elementary methods. However, up to now the standard method of deriving these relationships was to interpret $Z^{t, y, v}$ as the Malliavin derivative, or more precisely the Malliavin trace, of $Y^{t, y, v}$. Compared to the approach given in [9] this has the disadvantage that additional regularity assumptions which originate in the usage of the Malliavin calculus are needed. Nevertheless we want to outline how Malliavin calculus can be used to derive (33), thus giving a proof of (33) in this paper (though not in full generality). Since this approach entails variational derivatives of the forward processes $I$ and $\rho$ (see Equation (35)) we need the additional assumption that the coefficients $a$ and $g$ of the dynamics of $\rho$ have bounded derivatives.

Malliavin based proof of Lemma 3.9 under the additional assumptions that a and $g$ have bounded derivatives: Let $I_{t}^{n}$ be the solution of the SDE

$$
d I_{t}^{n}=I_{t}^{n}\left(\mu_{I}-r\right) d t+\sigma_{I} I_{t}^{n}\left(\left(1-\frac{1}{n}\right) \rho_{t} d W_{t}^{1}+\sqrt{1-\left(1-\frac{1}{n}\right)^{2} \rho^{2}} d W_{t}^{2}\right)
$$

It is straigtforward to show that $I_{T}^{n}$ converges to $I_{T}$ in $L^{2}$. By taking a subsequence, we may assume that $I_{T}^{n}$ converges to $I_{T}$ almost surely.

Next we approximate the payoff function $h$ by a sequence of everywhere differentiable and globally Lipschitz continuous functions. More precisely, let $\varphi(x)=\frac{1}{\sqrt{2 \pi}} e^{-\frac{x^{2}}{2}}$ the density of a standard normal distibution and let $\varphi^{n}(x)=n \varphi(n x)$ for all $n \geq 1$. We define $h^{n}$ as the convolution with $\varphi$, i.e. $h^{n}=h * \varphi^{n}$. Observe that $h^{n}$ is Lipschitz continuous with respect to the same Lipschitz constant as $h$. Note that Lipschitz continuity of $h$ implies uniform convergence of $h^{n}$ to $h$, hence $h^{n}\left(I_{T}^{n}\right)$ converges a.s. to $h\left(I_{T}\right)$. Moreover, $h^{n}$ is differentiable.

As before, we denote by $I_{s}^{n, t, y, v}$ the process $I_{s}^{n}$ conditioned on $I_{t}^{n}=y$ and $\rho_{t}=v$. We further define $\psi^{n}(t, y, v)=\tilde{\mathbb{E}}\left[h^{n}\left(I_{T}^{n, t, y, v}\right)\right]$ for all $n \geq 1$, where $\tilde{\mathbb{E}}$ denotes the expectation with respect to the measure $\tilde{\mathbb{P}}$ defined in Section 2. Note that by the same methods as in Section 3.3 it can be shown that the $\psi^{n}$ are differentiable; indeed, due to the factor $\left(1-\frac{1}{n}\right)$ the integrability condition (31) is trivial. Moreover, its derivatives are bounded, i.e. the $\psi^{n}$ are Lipschitz continuous.

We proceed by showing that $\psi^{n}$ converges pointwise to $\psi$. Indeed, with $L \in \mathbb{R}_{+}$being the 
Lipschitz constant of $h$, we have

$$
\begin{aligned}
\lim _{n}\left|\psi^{n}(t, y, v)-\psi(t, y, v)\right| & \leq \lim _{n} \sqrt{\tilde{\mathbb{E}}\left|h^{n}\left(I_{T}^{n, t, y, v}\right)-h\left(I_{T}^{t, y, v}\right)\right|^{2}} \\
& \leq \lim _{n} \sqrt{4\left(\tilde{\mathbb{E}}\left|h^{n}\left(I_{T}^{n, t, y, v}\right)-h\left(I_{T}^{n, t, y, v}\right)\right|^{2}+\tilde{\mathbb{E}}\left|h\left(I_{T}^{n, t, y, v}\right)-h\left(I_{T}^{t, y, v}\right)\right|^{2}\right)} \\
& \leq \lim _{n} \sqrt{4\left(\left\|h^{n}-h\right\|_{\infty}+L^{2} \tilde{\mathbb{E}}\left|I_{T}^{n}-I_{T}\right|^{2}\right)}=0 .
\end{aligned}
$$

Let $\left(Y^{n, t, y, v}, Z^{n, t, y, v}\right)$ be the solution of the BSDE

$$
Y_{s}^{n, t, y, v}=h^{n}\left(I_{T}^{n, t, y, v}\right)-\int_{s}^{T} Z_{u}^{n, t, y, v} d W_{u}-\int_{s}^{T} Z_{u}^{n, 1, t, y, v} \frac{\mu_{X}-r}{\sigma_{X}} d u,
$$

for $s \in[t, T]$. Since $h^{n}\left(I_{T}^{n, t, y, v}\right)$ converges to $h\left(I_{T}^{t, y, v}\right)$ in $L^{2}(\mathbb{P})$, standard a priori estimates for Lipschitz BSDEs, or simply the Ito isometry under the measure $\tilde{\mathbb{P}}$, imply that $\left(Y^{n}, Z^{n}\right)$ converges to $(Y, Z)$ in $\mathbb{S}(\mathbb{R}) \otimes \mathbb{H}_{T}^{2}\left(\mathbb{R}^{3}\right)$.

Notice that, due to the Markov property, we have $Y_{s}^{n, t, y, v}=\tilde{\mathbb{E}}\left[h^{n}\left(I_{T}^{n, t, y, v}\right) \mid \mathcal{F}_{s}\right]=\psi^{n}\left(s, I_{s}^{n, t, y, v}, \rho_{s}^{t, v}\right)$. Since the approximations $\psi^{n}$ are Lipschitz continuous, we may apply the chain rule, which yields

$$
D_{u} Y_{s}^{n, t, y, v}=\partial_{y} \psi^{n}\left(s, I_{s}^{n, t, y, v}, \rho_{s}^{t, v}\right) D_{u} I_{s}^{n, t, y, v}+\partial_{v} \psi^{n}\left(s, I_{s}^{n, t, y, v}, \rho_{s}^{t, v}\right) D_{u} \rho_{s}^{t, v}, \quad u \in[t, T],
$$

where $D_{u}$ denotes the Malliavin derivative of $Y^{n, t, y, v}, I^{n, t, y, v}$ and $\rho^{t, v}$ respectively. $D_{u} I^{n, t, y, v}$ and $D_{u} \rho^{t, v}$ are solutions of linear SDEs (see Theorem 2.2.1 in [12]). In particular this guarantees right continuity of $D_{u} Y_{s}^{n, t, y, v}$ in $s$.

By the Clark-Ocone formula, the process $Z^{n, t, y, v}$ is the predictable projection of $h^{n}\left(I_{T}^{n, t, y, v}\right)$ under the measure $\tilde{P}$. More precisely, for all $s \in[t, T]$, we have $Y_{s}^{n, t, y, v}=\int_{t}^{s} \tilde{\mathbb{E}}\left[D_{u} Y_{s}^{n, t, y, v} \mid \mathcal{F}_{u}\right] d \tilde{W}_{u}$, where $\tilde{W}_{t}=\left(W_{t}^{1}+\frac{\mu_{X}-r}{\sigma_{X}} t, W_{t}^{2}, W_{t}^{3}\right)$ and $\tilde{\mathbb{E}}\left[\cdot \mid \mathcal{F}_{u}\right]$ stands for the predictable projection operator with respect to $\tilde{\mathbb{P}}$. Due to the right continuity of $D_{u} Y_{s}^{n, t, y, v}$ in $s$, we may interchange the Malliavin and the predictable projection operator, which yields

$$
Z_{u}^{n, t, y, v}=\tilde{\mathbb{E}}\left[D_{u} Y_{s}^{n, t, y, v} \mid \mathcal{F}_{u}\right]=\lim _{s \downarrow u} D_{u} Y_{s}^{n, t, y, v}=\sigma^{n}\left(u, I_{u}^{n, t, y, v}, \rho_{u}^{t, v}\right)^{*}\left(\begin{array}{c}
\partial_{y} \psi^{n}\left(u, I_{u}^{n, t, y, v}, \rho_{u}^{t, v}\right) \\
\partial_{v} \psi^{n}\left(u, I_{u}^{n, t, y, v}, \rho_{u}^{t, v}\right)
\end{array}\right),
$$

where

$$
\sigma^{n}(y, v)=\left(\begin{array}{ccc}
y \sigma_{I}\left(1-\frac{1}{n}\right) v & y \sigma_{I} \sqrt{1-\left(1-\frac{1}{n}\right)^{2} v^{2}} & 0 \\
g(v) \gamma & g(v) \delta & g(v) \sqrt{1-\gamma^{2}-\delta^{2}}
\end{array}\right) .
$$

We next show that the partial derivatives $\partial_{y} \psi^{n}$ and $\partial_{v} \psi^{n}$ converge pointwise to $\partial_{y} \psi$ and $\partial_{v} \psi$, respectively. To this end denote again the derivatives of $I_{t}^{n, t, y, v}$ with respect to $v$ by $\bar{I}_{t}^{n, t, y, v}$. Lemma 3.7 yields that $\sup _{n} \mathbb{E}\left|\bar{I}_{T}^{n}\right|^{p}<\infty$, which further implies that the sequence $\left|\bar{I}_{T}^{n}\right|$ is uniformly integrable. Moreover,

$$
\begin{aligned}
& \left|\partial_{v} \psi^{n}-\partial_{v} \psi\right| \\
\leq & \tilde{\mathbb{E}}\left|\left(h^{n}\right)^{\prime}\left(I_{T}^{n, t, y, v}\right) \bar{I}_{T}^{n, t, y, v}-h^{\prime}\left(I_{T}^{t, y, v}\right) \bar{I}_{T}^{t, y, v}\right| \\
\leq & \tilde{\mathbb{E}}\left|\left(h^{n}\right)^{\prime}\left(I_{T}^{n, t, y, v}\right)\right|\left|\bar{I}_{T}^{n, t, y, v}-\bar{I}_{T}^{t, y, v}\right|+\tilde{\mathbb{E}}\left|\bar{I}_{T}^{t, y, v}\right|\left|\left(h^{n}\right)^{\prime}\left(I_{T}^{n, t, y, v}\right)-h^{\prime}\left(I_{T}^{t, y, v}\right)\right| .
\end{aligned}
$$


We show separately that both summands of (38) converge to 0 as $n \rightarrow \infty$. Since the approximating functions $h^{n}$ have one common Lipschitz constant $L \in \mathbb{R}_{+}$, the derivatives satisfy $\left|\left(h^{n}\right)^{\prime}\right| \leq L$ for all $n \geq 1$. Consequently, $\lim _{n} \tilde{\mathbb{E}}\left|\left(h^{n}\right)^{\prime}\left(I_{T}^{n, t, y, v}\right)\right|\left|\bar{I}_{T}^{n, t, y, v}-\bar{I}_{T}^{t, y, v}\right| \leq L \lim _{n} \tilde{\mathbb{E}}_{T} \bar{I}_{T}^{n, t, y, v}-\bar{I}_{T}^{t, y, v} \mid$. Next, let $\tau_{k}=T \wedge \inf \left\{t \geq 0:\left|\rho_{t}\right|=\left(1-\frac{1}{k}\right)\right\}$ for all $k \geq 1$. Then the stopped processes $\bar{I}_{t \wedge \tau_{k}}^{n}$ converge to $\bar{I}_{t \wedge \tau_{k}}$ in $L^{2}$ as $n \rightarrow \infty$ (see f.ex. Sec. 4, Ch.V, [13]). Therefore, by dominated convergence, for every $k \geq 1$ we have

$$
\begin{aligned}
\lim _{n} \tilde{\mathbb{E}}\left|\bar{I}_{T}^{n, t, y, v}-\bar{I}_{T}^{t, y, v}\right| & \leq \lim _{n}\left(\tilde{\mathbb{E}}\left|1_{\left\{\tau_{k}<T\right\}}\left(\bar{I}_{T}^{n, t, y, v}-\bar{I}_{T}^{t, y, v}\right)\right|+\tilde{\mathbb{E}}\left|1_{\left\{\tau_{k} \geq T\right\}}\left(\bar{I}_{T}^{n, t, y, v}-\bar{I}_{T}^{t, y, v}\right)\right|\right) \\
& \leq \tilde{\mathbb{E}}\left(1_{\left\{\tau_{k}<T\right\}}\left(\left|\bar{I}_{T}^{n, t, y, v}\right|+\tilde{\mathbb{E}}\left|\bar{I}_{T}^{t, y, v}\right|\right)\right) .
\end{aligned}
$$

Recall that $\bar{I}_{t}^{n}$ is uniformly integrable, and that $\lim _{k} \tilde{P}\left(\tau_{k}=T\right)=1$; hence by letting $k \rightarrow \infty$ we get that $\lim _{n} \tilde{\mathbb{E}}\left|\bar{I}_{T}^{n, t, y, v}-\bar{I}_{T}^{t, y, v}\right|=0$, and hence the first summand in (38) converges to 0 .

In order to show that the second summand in (38) vanishes, we first show that, for $x_{n} \rightarrow x$, $\left(h^{n}\right)^{\prime}\left(x_{n}\right) \rightarrow h^{\prime}(x)$. If $x$ is a point of continuity of $h^{\prime}$, then we have the following estimate

$$
\begin{aligned}
\left|\left(h^{n}\right)^{\prime}\left(x_{n}\right)-h^{\prime}(x)\right| & =\left|\int \varphi_{n}\left(x_{n}-y\right) h^{\prime}(y) d y-h^{\prime}(x)\right| \\
& =\left|\int \varphi_{n}\left(x_{n}-x+x-y\right) h^{\prime}(y) d y-h^{\prime}(x)\right| \\
& =\left|\int \varphi_{n}(x-z) h^{\prime}\left(z+x_{n}-x\right) d z-h^{\prime}(x)\right| \quad\left(z:=y-x_{n}+x\right) \\
& \leq\left|\int \varphi_{n}(x-z) h^{\prime}(z) d z-h^{\prime}(x)\right|+\left|\int \varphi_{n}(x-z)\left[h^{\prime}\left(z+x_{n}-x\right)-h^{\prime}(z)\right] d z\right| .
\end{aligned}
$$

Applying the transformation $y:=n(x-z)$ in each term on the right hand side of the inequality, together with dominated convergence and the continuity of $h^{\prime}$ in $x$, yields that $\lim _{n} h^{\prime}\left(x_{n}\right)=$ $h^{\prime}(x)$.

Since $I_{T}$ has a density, $\left(h^{n}\right)^{\prime}\left(I_{T}^{n, t, y, v}\right)$ converges to $h^{\prime}\left(I_{T}^{t, y, v}\right)$ almost everywhere. Consequently, by dominated convergence, we obtain $\lim _{n} \tilde{\mathbb{E}}\left|I_{T}^{t, y, v}\right|\left|\left(h^{n}\right)^{\prime}\left(I_{T}^{n, t, y, v}\right)-h^{\prime}\left(I_{T}^{t, y, v}\right)\right|=0$. Thus we have shown that $\lim _{n} \partial_{v} \psi^{n}(t, y, v)=\partial_{v} \psi(t, y, v)$ for all $t \in[0, T], y \in \mathbb{R}$ and $v \in(-1,1)$.

Notice that $I_{s}^{n, t, y, v}=y I_{s}^{n, t, 1, v}$ and $\bar{I}_{s}^{n, t, y, v}=y \bar{I}_{s}^{n, t, 1, v}$, which implies that for all $t \geq 0$ and $v \in(-1,1)$, the sequence $\partial_{v} \psi^{n}(t, \cdot, v)$ converges to $\partial_{v} \psi(t, \cdot, v)$ uniformly in $y$ on all compact sets of $\mathbb{R}$. Similarly, one can show locally uniform convergence in $y$ of the partial derivatives $\partial_{y} \psi^{n}$ to $\partial_{y} \psi$.

This finally yields that $\partial_{v} \psi^{n}\left(s, I_{s}^{n, t, y, v}, \rho_{s}^{t, v}\right)$ converges to $\partial_{v} \psi\left(s, I_{s}^{t, y, v}, \rho_{s}^{t, v}\right)$, and $\partial_{y} \psi^{n}\left(s, I_{s}^{n, t, y, v}, \rho_{s}^{t, v}\right)$ to $\partial_{y} \psi\left(s, I_{s}^{t, y, v}, \rho_{s}^{t, v}\right)$, almost surely. Moreover, by combining this with Equation (36), we get that $Z^{n}$ converges almost surely to $\sigma\left(s, I_{s}^{t, y, v}, \rho_{s}^{t, v}\right)^{*}\left(\begin{array}{c}\partial_{y} \psi\left(s, I_{s}^{t, y, v}, \rho_{s}^{t, v}\right) \\ \partial_{v} \psi\left(s, I_{s}^{t, y, v}, \rho_{s}^{t, v}\right)\end{array}\right)$. Since $Z^{n}$ converges also to $Z$ in $\mathbb{H}^{2}$, we obtain the result. 


\section{A class of correlation dynamics which fulfill the main assump- tions}

In this part of the work we characterize a class of dynamics which fulfill Conditions (H1) and (H2). The result has already been mentioned as Theorem 2.5 in Section 2 above. For ease of reference we state it again below.

Moreover we will give an extension of Theorem 2.5 in Proposition 4.3. We will see in the example section, that this extension enables us to show that the so-called Jacobi processes also fit into our framework. In contrast to the dynamics given in Theorem 2.5 the diffusion coefficient of a Jacobi processes has unbounded derivatives in -1 and 1 .

We first collect some notation and facts on attainability of boundaries for diffusions. The material is taken from [10]. Suppose we are given a general diffusion

$$
d X_{t}=\mu\left(X_{t}\right) d t+\sigma\left(X_{t}\right) d W_{t}, \quad l \leq X_{0} \leq r,
$$

where $l$ (resp. $r$ ) denote the left boundary $l$ (resp. the right boundary). In the following we only consider the analysis for the left boundary. We define, for $x \in(l, r)$,

$$
\begin{aligned}
s(v) & =\exp \left(-\int_{v_{0}}^{v} \frac{2 \mu(w)}{\sigma^{2}(w)} d w\right), \quad v_{0} \in(l, x), \\
S(x) & =\int_{x_{0}}^{x} s(v) d v, \quad x_{0} \in(l, x), \\
S[c, d] & =S(d)-S(c), \quad(c, d) \in(l, r), \\
S(l, x] & =\lim _{c \rightarrow l} S[c, x] .
\end{aligned}
$$

We already indicate that $x_{0}$ and $v_{0}$ will be of no relevance in the following. $S$ is called the scale measure whereas $M$ is the speed measure:

$$
\begin{aligned}
m(x) & =\frac{1}{\sigma^{2}(x) s(x)}, \\
M[c, d] & =\int_{c}^{d} m(x) d x .
\end{aligned}
$$

We also need

$$
\Sigma(l)=\lim _{c \rightarrow l} \int_{c}^{x} M[v, x] d S(v) .
$$

According to [10] the boundary $l$ is attracting if $S(l, x]<\infty$ and this criterion applies independently of $x \in(l, r)$. Moreover the boundary $l$ is said to be

1. attainable if $\Sigma(l)<\infty$,

2. unattainable if $\Sigma(l)=\infty$.

For a proof of the following Lemma see Chapter 15.6 in [10]. 
Lemma 4.1. $S(l, x]=\infty$ implies $\Sigma(l)=\infty$.

With this at hand, we can find sufficient conditions on the coefficients of the correlation dynamics so that condition (H1) is satisfied. Let again $\rho^{t, v}$ and $\bar{\rho}^{t, v}$ be defined as in the SDEs (8) and (9). To simplify notation, from now on we will suppress the dependence on $t$ and $v$ and only write $\rho$ resp. $\bar{\rho}$.

Lemma 4.2. Let $a$ and $g$ be continuously differentiable. We assume that $g$ does not have any roots in $(-1,1)$. If

$$
\underset{x \uparrow 1}{\limsup } \frac{2 a(x)(1-x)}{g^{2}(x)}<0 \text { and } \liminf _{x \downarrow-1} \frac{2 a(x)(1+x)}{g^{2}(x)}>0,
$$

then Condition (H1) is satisfied.

Proof. We show that $\rho$ does not reach -1 . By Condition (39) there exist $\varepsilon>0, \delta>0$ and $v_{0} \in(-1,1)$ such that $\frac{2 a(w)}{g^{2}(w)} \geq \frac{\varepsilon}{1+w}$, for all $-1<w<v_{0}<-1+\delta$. Hence

$$
s(v)=\exp \left(\int_{v}^{v_{0}} \frac{2 a(w)}{g^{2}(w)} d w\right) \geq \exp \left(\int_{v}^{v_{0}} \frac{\varepsilon}{1+w} d w\right)=C \exp (-\log (1+v))=C \frac{1}{1+v} .
$$

Hence

$$
S[c, x] \geq C \int_{c}^{x} \frac{1}{1+v} d v \longrightarrow \infty,
$$

for $c \rightarrow-1$, i.e. by Lemma 4.1 we obtain that $\rho$ does not reach -1 . We treat the boundary 1 similarly and hence get Assumption (H1).

The next proposition provides conditions under which Condition (H2) is satisfied. We will need two auxiliary processes $\tilde{a}$ and $\tilde{g}$ defined by

$$
\begin{aligned}
& \tilde{a}_{u}=\frac{2 \rho_{u}}{\left(1-\rho_{u}^{2}\right)} g\left(\rho_{u}\right)+2 g^{\prime}\left(\rho_{u}\right), \text { and } \\
& \tilde{g}_{u}=\frac{2 \rho_{u}}{\left(1-\rho_{u}^{2}\right)} a\left(\rho_{u}\right)+2 a^{\prime}\left(\rho_{u}\right)+\frac{g^{2}\left(\rho_{u}\right)}{1-\rho_{u}^{2}}+\frac{4 \rho_{u}^{2} g^{2}\left(\rho_{u}\right)}{\left(1-\rho_{u}^{2}\right)^{2}}+\left(g^{\prime}\left(\rho_{u}\right)\right)^{2}+\frac{4 \rho_{u}}{1-\rho_{u}^{2}} g\left(\rho_{u}\right) g^{\prime}\left(\rho_{u}\right) .
\end{aligned}
$$

Proposition 4.3. Assume the conditions of Lemma 4.2 are satisfied. Then Assumption (H1) holds, and therefore, $\tilde{a}$ and $\tilde{g}$ are well defined. Suppose $\tilde{a}$ is bounded and $\tilde{g}$ is bounded from above. Then Assumption (H2) is satisfied, and hence, the delta hedge is given as in Theorem 2.1.

Proof. We start by an application of Ito's formula on the process $\Phi$ defined by $\Phi_{s}=f\left(\rho_{s}, \bar{\rho}_{s}\right)$, where $f$ is given by $f(x, y)=\frac{y^{2}}{1-x^{2}}$. Note that $f_{x}(x, y)=\frac{2 x y^{2}}{\left(1-x^{2}\right)^{2}}, f_{x x}=\frac{2 y^{2}}{\left(1-x^{2}\right)^{2}}+\frac{8 x^{2} y^{2}}{\left(1-x^{2}\right)^{3}}$, 


$$
\begin{aligned}
f_{y}(x, y) & =\frac{2 y}{1-x^{2}}, f_{y y}=\frac{2}{1-x^{2}} \text { and } f_{x y}=\frac{4 x y}{\left(1-x^{2}\right)^{2}} \text {. We have } \\
\Phi_{s}= & \Phi_{t}+\int_{t}^{s} \frac{2 \rho_{u} \bar{\rho}_{u}^{2}}{\left(1-\rho_{u}^{2}\right)^{2}}\left[a\left(\rho_{u}\right) d u+g\left(\rho_{u}\right) d \hat{W}_{u}\right]+\int_{t}^{s} \frac{2 \bar{\rho}_{u}}{1-\rho_{u}^{2}}\left[a^{\prime}\left(\rho_{u}\right) \bar{\rho}_{u} d u+g^{\prime}\left(\rho_{u}\right) \bar{\rho}_{u} d \hat{W}_{u}\right] \\
& +\frac{1}{2} \int_{t}^{s}\left[\frac{2 \bar{\rho}_{u}^{2}}{\left(1-\rho_{u}^{2}\right)^{2}}+\frac{8 \rho_{u}^{2} \bar{\rho}_{u}^{2}}{\left(1-\rho^{2}\right)^{3}}\right] g^{2}\left(\rho_{u}\right) d u+\frac{1}{2} \int_{t}^{s} \frac{2}{1-\rho_{u}^{2}}\left(g^{\prime}\left(\rho_{u}\right)\right)^{2} \bar{\rho}_{u}^{2} d u \\
& +\int_{t}^{s} \frac{4 \rho_{u} \bar{\rho}_{u}}{\left(1-\rho^{2}\right)^{2}} g\left(\rho_{u}\right) g^{\prime}\left(\rho_{u}\right) \bar{\rho}_{u} d u \\
= & \Phi_{t}+\int_{t}^{s} \Phi_{u}\left[\frac{2 \rho_{u}}{\left(1-\rho_{u}^{2}\right)} g\left(\rho_{u}\right)+2 g^{\prime}\left(\rho_{u}\right)\right] d \hat{W}_{u} \\
& +\int_{t}^{s} \Phi_{u}\left[\frac{2 \rho_{u}}{\left(1-\rho_{u}^{2}\right)} a\left(\rho_{u}\right)+2 a^{\prime}\left(\rho_{u}\right)+\frac{g^{2}\left(\rho_{u}\right)}{1-\rho_{u}^{2}}+\frac{4 \rho_{u}^{2} g^{2}\left(\rho_{u}\right)}{\left(1-\rho_{u}^{2}\right)^{2}}+\left(g^{\prime}\left(\rho_{u}\right)\right)^{2}+\frac{4 \rho_{u}}{1-\rho_{u}^{2}} g\left(\rho_{u}\right) g^{\prime}\left(\rho_{u}\right)\right] d u .
\end{aligned}
$$

Thus, $\Phi$ is the solution of a linear stochastic equation and given by

$$
\Phi_{s}=\Phi_{t} \mathcal{E}\left(\int_{t}^{s} \tilde{a}_{u} d \hat{W}_{u}+\int_{t}^{s} \tilde{g}_{u} d u\right)
$$

Hence by our assumptions on $\tilde{a}$ and $\tilde{g}$, and by Lemma 3.5, all moments of $\sup _{t \leq u \leq T} \Phi_{u}$ are finite, which further yields Assumption (H2).

We use the two preceeding statements to prove Theorem 2.5.

Theorem 2.5. Let $a$ and $g$ be continuously differentiable with bounded derivatives. We assume that $g(-1)=g(1)=0$, and that $g$ does not have any roots in $(-1,1)$. If

$$
\limsup _{x \uparrow 1} \frac{2 a(x)(1-x)}{g^{2}(x)}<0 \text { and } \liminf _{x \downarrow-1} \frac{2 a(x)(1+x)}{g^{2}(x)}>0,
$$

then Conditions (H1) and (H2) are satisfied, and hence, the delta hedge is given as in Theorem 2.1.

Proof. Condition (H1) follows from Lemma 4.2. Since 1 and -1 are roots of $g$ we can write $\frac{g(x)}{1-x^{2}}=\frac{1}{1+x} \frac{g(x)-g(1)}{1-x}=\frac{-1}{1+x} \frac{g(x)-g(1)}{x-1}$ and hence $\frac{g(x)}{1-x^{2}}$ is bounded for $x \nearrow 1$ by the derivative of $g$ at $x=1$. Similarly for $x \searrow-1$, i.e. the fraction $\frac{g(x)}{1-x^{2}}$ is bounded on $[-1,1]$. Moreover, Condition (39) implies that there exists an $\varepsilon \in(0,1)$ such that all $x \in(-1,1)$ with $|x| \geq 1-\varepsilon$ satisfy $x a(x)<0$. Hence $\tilde{a}$ (resp. $\tilde{g}$ ) is bounded (resp. bounded from above) and therefore we obtain the result by Proposition 4.3.

Remark 4.4. 1. Note that the conditions on the coefficients $a$ and $g$ of the correlation dynamics in Theorem 2.5 are more restrictive than in Proposition 4.3. This is mainly for ease of exposition in Section 2. In Section 5.1 an example is given where the coefficient $g$ of the correlation dynamics does not have a bounded derivative.

2. It is possible to prove Theorem 2.5 without considering the auxiliary processes $\tilde{a}$ and $\tilde{g}$ and using Proposition 4.3. In the following we give a rough sketch of a more intuitive proof of 
Theorem 2.5. That alternative proof consists in showing that all moments of the process $Y_{t}=\frac{1}{1-\rho_{t}^{2}}$ are finite, from which one easily deduces Condition (H2) to be satisfied. From Ito's formula we obtain

$$
d Y_{t}=\frac{2 \rho_{t}}{\left(1-\rho_{t}^{2}\right)} g\left(\rho_{t}\right) Y_{t} d \hat{W}_{t}+2 \rho_{t} a\left(\rho_{t}\right) Y_{t}^{2} d t+\left(1+3 \rho_{t}^{2}\right) \frac{g^{2}\left(\rho_{t}\right)}{\left(1-\rho_{t}^{2}\right)^{2}} Y_{t} d t,
$$

showing that $Y$ is a linear SDE with an additional drift term growing quadratically in $Y$. Condition (15) implies that there exists an $\varepsilon \in(0,1)$ such that all $x \in(-1,1)$ with $|x| \geq 1-\varepsilon$ satisfy $x a(x)<0$. Moreover, $\left\{\left|\rho_{s}\right| \leq 1-\varepsilon\right\}=\left\{Y_{s} \leq \frac{1}{2 \varepsilon-\varepsilon^{2}}\right\}$, and consequently, the quadratic drift term in the dynamics of $Y$ has a shrinking effect as soon as $Y$ exceeds $C_{\varepsilon}=\frac{1}{2 \varepsilon-\varepsilon^{2}}$. In other words, $Y$ can be shown to be dominated by the SDE

$$
d \check{Y}_{t}=\frac{2 \rho_{t}}{\left(1-\rho_{t}^{2}\right)} g\left(\rho_{t}\right) \check{Y}_{t} d \hat{W}_{t}+\left(1+3 \rho_{t}^{2}\right) \frac{g^{2}\left(\rho_{t}\right)}{\left(1-\rho_{t}^{2}\right)^{2}} \check{Y}_{t} d t+C_{\varepsilon} d t
$$

that, by standard arguments, can be shown to possess finite moments.

\section{$5 \quad$ Examples}

The aim of this final section is to give some explicit correlation dynamics which fall within the framework above. We start by modelling correlation processes directly as solutions of various SDEs with values in $[-1,1]$ in Subsection 5.1. Another approach is used in Subsection 5.2 where we use mappings of an Ornstein-Uhlenbeck process onto the open intervall $(-1,1)$.

\subsection{Modelling correlation directly}

Example 5.1. Of course all processes that are bounded away from -1 and 1 also fulfill the Conditions (H1) and (H2).

Example 5.2. For $a(x)=\kappa(\vartheta-x)$, with $\kappa>0, \vartheta \in(-1,1)$, and $g(x)=\alpha\left(1-x^{2}\right)$ in the dynamics of $\rho$, the prerequisites of Theorem 2.5 are fulfilled.

Example 5.3. Let $a$ and $g$ be polynomials. Assume that $g(-1)=g(1)=0$, and that $g$ does not have any roots in $(-1,1)$. If

$$
\lim _{x \uparrow 1} \frac{a(x)}{g^{2}(x)}=-\infty \text { and } \lim _{x \downarrow-1} \frac{a(x)}{g^{2}(x)}=+\infty,
$$

then the prerequisites of Theorem 2.5 are satisfied.

The common denominator of the precceding two examples is that the coefficients in the dynamics of $\rho$ fulfill the prerequisites of Theorem 2.5, which includes bounded derivatives. We now want to give an example where $g$ does not have bounded derivatives in -1 and 1 . We consider so-called Jacobi processes, which are given by the solution of

$$
\rho_{s}^{t, v}=v+\int_{t}^{s} \kappa\left(\vartheta-\rho_{u}^{t, v}\right) d u+\int_{t}^{s} \alpha \sqrt{\left(1-\left(\rho_{s}^{t, v}\right)^{2}\right.} \hat{W}_{u} .
$$


Jacobi processes might be of interest for modelling stochastic correlation, because their stationary and transitional densities are well known and can be obtained quite explicit, see for example $[2]$.

By exploiting the boundary theory at the beginning of Section 4 or by checking when Condition (15) holds one can easily show that for $\kappa, \alpha>0$ and $\vartheta$ such that

$$
\kappa \geq \frac{\alpha^{2}}{1 \pm \vartheta}
$$

the boundaries -1 and 1 of the process defined in (43) are unattainable. Hence, we have that Assumption (H1) is fulfilled. We want to apply Proposition 4.3 and therefore have to check the boundedness of $\tilde{a}$ and upper boundedness of $\tilde{g}$. Note that $\tilde{g}$ turns into

$$
\begin{aligned}
\tilde{g}_{u} & =\frac{2 \rho_{u}}{\left(1-\rho_{u}^{2}\right)} a\left(\rho_{u}\right)+2 a^{\prime}\left(\rho_{u}\right)+\frac{\alpha^{2}}{1-\rho_{u}^{2}} \\
& =\frac{2 \rho_{u}}{\left(1-\rho_{u}^{2}\right)}\left(\kappa\left(\vartheta-\rho_{u}\right)\right)-2 \kappa+\frac{\alpha^{2}}{1-\rho_{u}^{2}},
\end{aligned}
$$

and $\tilde{a}_{u}=0$. In order to ensure upper boundedness of $\tilde{g}_{u}$ it is sufficient to show the existence of an $\varepsilon>0$ such that $2 \rho_{u}\left(\kappa\left(\vartheta-\rho_{u}\right)\right)+\alpha^{2}<0$, for all $\left|\rho_{u}\right|>1-\varepsilon$, P-a.s. This is guaranteed by choosing $\kappa, \alpha>0$ and $\vartheta$ such that the constants $\rho_{(1)}$ and $\rho_{(2)}$ defined by

$$
\rho_{(1),(2)}=\frac{\vartheta}{2} \pm \sqrt{\frac{\vartheta^{2}}{4}+\frac{\alpha^{2}}{2 \kappa}}
$$

fulfill

$$
-1<\rho_{(1)} \leq \rho_{(2)}<1 .
$$

Note, for example, that for $\alpha=1$ and $\vartheta=0.9$ Condition (44) is satisfied by $\kappa=10$ and that this choice of parameters also fulfills (45).

\subsection{Modelling correlation with Ornstein-Uhlenbeck processes}

In the previous section we assumed that the stochastic correlation process is described in terms of the SDE (6). The correlation dynamics need not to be modelled directly. Alternatively, one can use a continuous bijection $b:(-1,1) \rightarrow \mathbb{R}$, and model at first place the transformed process $b\left(\rho_{t}\right)$ as an SDE. This has the advantage that $b\left(\rho_{t}\right)$ can be modelled as a diffusion on $\mathbb{R}$ with Lipschitz coefficients. The correlation may be modelled as a standard mean reverting process, for example an Ornstein-Uhlenbeck process, the dynamics of which can be calibrated via standard methods.

In this section we will discuss this alternative approach of modelling correlation. As a paradigma example we will choose as bijection $b(x)=\frac{x}{\sqrt{1-x^{2}}}$, and we will assume that $U_{t}=b\left(\rho_{t}\right)$ is an Ornstein-Uhlenbeck process with dynamics

$$
d U_{t}=a\left(\vartheta-U_{t}\right) d t+\sigma_{U} d\left(\gamma d W_{t}^{1}+\delta d W_{t}^{2}+\sqrt{1-\gamma^{2}-\delta^{2}} d W_{t}^{3}\right),
$$


where $a>0, \vartheta \in \mathbb{R}, \sigma_{U}>0$ and $\gamma, \delta \in(-1,1)$ are such that $\gamma^{2}+\delta^{2} \leq 1$. Notice that $\rho_{t}=\frac{U_{t}}{\sqrt{1+U_{t}^{2}}}$. We will prove that the prerequisites of Theorem 2.1 are satisfied and hence that the local risk minimization strategy is defined as in (12).

Lemma 5.4. The correlation process $\rho_{t}$ satisfies Conditions (H1) and (H2) and hence Theorem 2.1 holds in this setting.

Proof. The proof is a simple application of Ito's formula. The first and the second derivative of $\left.b^{-1}: \mathbb{R} \rightarrow\right]-1,1\left[, x \mapsto \frac{x}{\sqrt{1+x^{2}}}\right.$ are given by $\left(b^{-1}(x)\right)^{\prime}=\left(1+x^{2}\right)^{-\frac{3}{2}}$ and $\left(b^{-1}(x)\right)^{\prime \prime}=-3 x\left(1+x^{2}\right)^{-\frac{5}{2}}$. Again we set $\hat{W}_{t}=\gamma W_{t}^{1}+\delta W_{t}^{2}+\sqrt{1-\gamma^{2}-\delta^{2}} W_{t}^{3}$. We obtain

$$
d \rho_{t}=\left(1-\rho_{t}^{2}\right)^{\frac{3}{2}} \sigma_{U} d \hat{W}_{t}+\left(1-\rho_{t}^{2}\right)\left(a \vartheta\left(1-\rho_{t}^{2}\right)^{\frac{1}{2}}-a \rho_{t}-\frac{3}{2} \rho_{t}\left(1-\rho_{t}^{2}\right) \sigma_{U}^{2}\right) d t
$$

It is straightforward to show that the coefficients of the SDE (47) satisfy the conditions of Theorem 2.5. Hence the result.

\section{References}

[1] S. Ankirchner, P. Imkeller, and G. Reis. Pricing and hedging of derivatives based on nontradeable underlyings. To appear in Mathematical Finance, 2009.

[2] C. Boortz. Modelling correlation risk. Master thesis. Technische Universität Berlin, 2008.

[3] M. H. A. Davis. Optimal hedging with basis risk. In From stochastic calculus to mathematical finance, pages 169-187. Springer, Berlin, 2006.

[4] R. Durrett. Probability: theory and examples. Duxbury Press, Belmont, CA, second edition, 1996.

[5] N. El Karoui, S. Peng, and M. C. Quenez. Backward stochastic differential equations in finance. Math. Finance, 7(1):1-71, 1997.

[6] C. Frei and M. Schweizer. Exponential utility indifference valuation in two Brownian settings with stochastic correlation. Adv. in Appl. Probab., 40(2):401-423, 2008.

[7] V. Henderson. Valuation of claims on nontraded assets using utility maximization. Math. Finance, 12(4):351-373, 2002.

[8] H. Hulley and T. A. McWalter. Quadratic hedging of basis risk. Research Paper Series 225, Quantitative Finance Research Centre, University of Technology, Sydney, June 2008.

[9] P. Imkeller, A. Réveillac, and A. Richter. Differentiability of quadratic BSDEs generated by continuous martingales and hedging in incomplete markets. arxiv: 0907.0941. 
[10] S. Karlin and H. M. Taylor. A second course in stochastic processes. Academic Press Inc. [Harcourt Brace Jovanovich Publishers], New York, 1981.

[11] M. Monoyios. Performance of utility-based strategies for hedging basis risk. Quant. Finance, $4(3): 245-255,2004$.

[12] D. Nualart. The Malliavin calculus and related topics. Probability and its Applications (New York). Springer-Verlag, Berlin, second edition, 2006.

[13] P. E. Protter. Stochastic integration and differential equations, volume 21 of Applications of Mathematics (New York). Springer-Verlag, Berlin, second edition, 2004. Stochastic Modelling and Applied Probability.

[14] D. Revuz and M. Yor. Continuous martingales and Brownian motion, volume 293 of Grundlehren der Mathematischen Wissenschaften [Fundamental Principles of Mathematical Sciences]. Springer-Verlag, Berlin, third edition, 1999.

[15] M. Schweizer. A guided tour through quadratic hedging approaches. In Option pricing, interest rates and risk management, Handb. Math. Finance, pages 538-574. Cambridge Univ. Press, Cambridge, 2001.

[16] M. Schweizer. Local risk-minimization for multidimensional assets and payment streams. In Advances in Mathematics of Finance, pages 213-229. Banach Center Publications (83), 2008.

[17] C. van Emmerich. Modelling correlation as a stochastic process. Technical report, Bergische Universität Wuppertal, March 2006. 